\title{
Insights into assembly of the macromolecular inflammasome complex
}

\begin{abstract}
Dramatic advances in our understanding of the ultrastructure of the inflammasome and the molecular interactions involved in its assembly have recently been made. The adaptor protein ASC has been proposed to display prion-like activity that results in the formation of filamentous structures in the cell. These filamentouos structures can subsequently become inflammatory themselves if released into the extracellular space and then phagocytosed. Various groups have now utilised a variety of microscopy and structural approaches in order to visualise components of, and indeed the entire, inflammasome in both endogenous and overexpression systems. In this brief review we draw upon these new pieces of work to describe how our understanding of the global structure of the inflammasome has progressed in light of these new observations. In particular we begin by providing an initial perspective on the possible formation of small circular, wheel-like, oligomers resembling apoptosomes. We then address the current view that inflammasomes result from the formation of a much larger complex which may involve polymeric filaments. We discuss how these developments fit with recent theories of inflammatory signalling, what questions these advances raise, and propose key areas for further investigation.
\end{abstract}

Keywords: ASC, filament, death domain, innate immunity, NLRP3, AIM2, NLRC4, NLR, CARD, caspase-1

DOI 10.2478/infl-2014-0009

Received September 23, 2014; accepted November 24, 2014

*Corresponding author: Tom P. Monie: Department of Veterinary Medicine, University of Cambridge, Madingley Road, Cambridge, CB3 0ES, UK, Email: tpm22@cam.ac.uk

Tom P. Monie, Joseph P. Boyle: Departments of Biochemistry and Veterinary Medicine, University of Cambridge, Cambridge, CB3 OES, UK

\section{The inflammasome}

The inflammasome is a multi-protein macromolecular signalling complex that utilises induced proximity [1] to induce caspase-1 activation and subsequent processing of the proinflammatory cytokines pro-interleukin (IL) $-1 \beta$ and pro-IL-18 to their active forms [2-4] (Box 1). Inflammasomes form as a result of activation of specific cytosolic pattern recognition receptors that include members of the nucleotide-binding domain, and leucinerich repeat containing receptor (NLR) family such as NLRP1 (NLR family, pyrin domain containing 1), NLRP3, NAIP (Neuronal apoptosis inhibitory protein), NLRC4 (NLR family, CARD (caspase recruitment domain) domain containing 4), as well as Pyrin and AIM2 (Absent in melanoma 2) (Box 1) (Figure 1A \& B). Inflammasomeforming receptors all contain N-terminal effector domains from the death domain superfamily (Box 1) (Figure 1A \& B). These effector domains are crucial for mediating the protein-protein contacts required for complex formation and downstream signalling and processing events. Prior to activation the receptors exist in an autoinhibited state in the resting cell. However, following activation the receptor undergoes conformational changes which result in protein aggregation and cause the effector domains to become available for further interactions, for example with the adaptor protein ASC (apoptosis-associated speck-like protein containing a CARD) . This results in the rapid formation of a large, up to two microns wide, 'speck'-like structure in the cytosol of the cell (Box 1). The overall composition of the speck remains something of an enigma, but it can be broadly described as consisting of the activated receptor, the adaptor protein ASC, and inflammatory caspases such as caspase-1, caspase-5 and caspase-8 [2-4] (Figure 1B). Formation of the speck is driven by rapid self-association of the adaptor protein ASC, most likely with the activated receptor serving as a nucleation centre [5]. In the case of the NLRC4 inflammasome this occurs less than five minutes after receptor activation [6]. Inflammasome formation is a central component of the cellular response to damage 
Box 1: Key definitions

Adaptor protein - A protein that links two other functional proteins in a multiprotein complex. In the case of the inflammasome ASC acts as an adaptor protein by mediating the recruitment of the caspases to the activated receptor. ASC possesses both pyrin and caspase recruitment domains so mediates these interactions via interactions between death domain family members.

Death domain - A helical protein fold commonly found mediating multiprotein interactions in large protein complexes formed in inflammatory and death signalling pathways. The death domain superfamily contains four members: the death domain, the death effector domin, the pyrin domain, and the caspase recruitment domain.

Effector domain - A protein domain found in the initiating receptor that is involved in mediating further protein-protein interactions to either aid the formation of a multiprotein complex, or initiate protein signalling or catalytic processing cascades. In the inflammasome the pyrin and caspase recruitment domains of the NLR or AIM2 proteins function as effector domains.

Induced Proximity - When certain catalytic proteins, such as caspases, are brought into close enough proximity through binding to other cellular proteins they are able to become activated either as a result of auto-proteolysis, or through proteolytic cleavage by a near neighbour.

Inflammasome forming receptors - Cytosolic pattern recognition receptors (such as NLRP1, NLRP3, NLRC4 and AIM2) that when activated lead to the recruitment of ASC, the formation of a large multiprotein complex (speck) and result in the activation of caspases which in turn process the inflammatory cytokine precursors pro-IL-1 $\beta$ and pro-IL-18.

Speck - Large macromolecular complex (upto 2 microns diameter) formed upon the activation of inflammasome forming receptors and driven by the oligomerisation of ASC.

A

\begin{tabular}{|c|c|c|c|c|c|}
\hline PYD & NBD & LRR & CARD & NLRP1 & \\
\hline PYD & NBD & LRR & NLRP3 & & Inflammasome \\
\hline CARD & NBD & LRR & NLRC4 & & \\
\hline PYD & HIN200 & & AIM2 & & \\
\hline CARD & NB-ARC & WD40 & Apaf-1/DARK & & Anontosome \\
\hline CARD & NB-ARC & & CED4 & & \\
\hline
\end{tabular}

B
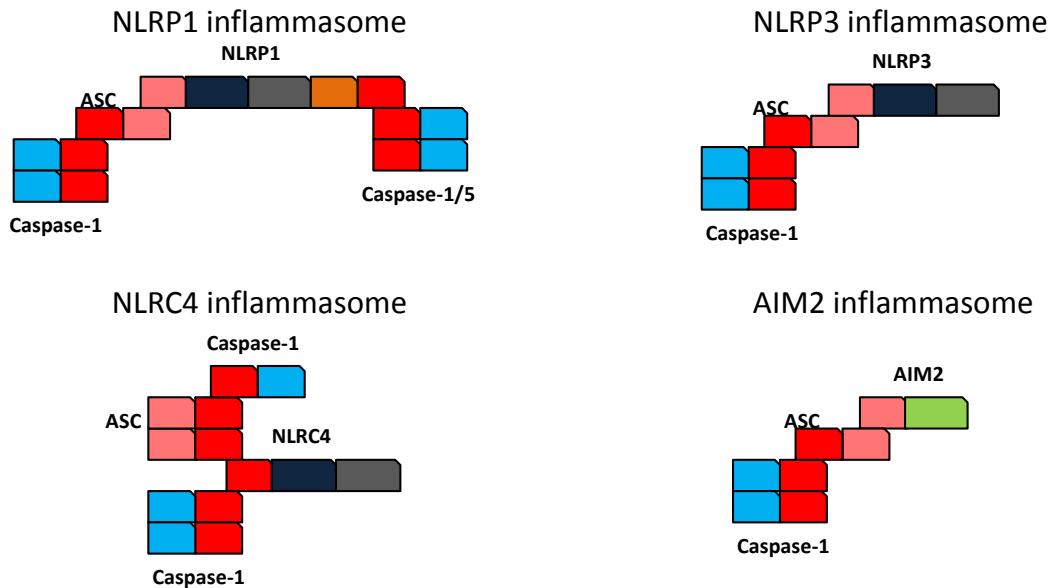

AIM2 inflammasome

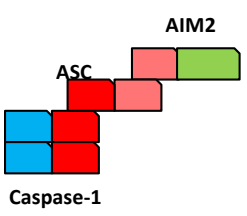

Figure 1: General architecture and organisation of the inflammasome. (A) Domain architecture of the major inflammasome forming pattern recognition receptors and apoptosome forming proteins from humans and Caenorhabditis elegans. (B) Schematic representation of the constituents of the NLRP1, NLRP3, NLRC4 and AIM2 inflammasomes. In both panels domains are coloured as follows: Pyrin (PYD) - pink; CARD - red; NBD /NB-ARC - blue, LRR/WD40 - grey. In addition in panel B the caspase catalytic domains are coloured cyan. Effector domains that mediate protein:protein interaction are are represented by the PYD and CARD domains. 
and threat. However, inappropriate and deregulated inflammasome activation can lead to the development of multiple diseases including gout, atherosclerosis and autoinflammatory disorders [7]. Of critical importance in inflammatory disease is the central role of the inflammasome in generating IL-1 $\beta$, which along with IL-1 $\alpha$, can be viewed as a master cytokine in the control and regulation of both local and systemic inflammation. This importance is exemplified by the success of the interleukin-1 blocking therapies anakinra, rilonacept and canakinumab for the treatment of chronic inflammatory conditions [8]. Consequently, a full understanding of the global inflammasome structure and how it functions will be an important part of delineating the aetiology of, and developing new treatments for, these diseases.

\section{Is the inflammasome structurally comparable to the apoptosome?}

Based on similarities between the domain organisation of the NLR family and Apaf-1 (Apoptotic protease-activating factor 1; Figure 1) it has long been assumed that the inflammasome will adopt a similar conformation to the apoptosis-inducing apoptosome formed by Apaf-1 and procaspase-9 and other apoptosome forming proteins [9] (Figure 2A). Electron Microscopy (EM) of purified preparations of NLRP1 and NLRC4/NAIP has shown these proteins to be capable of forming comparable in vitro wheel-like structures with an approximate diameter of between 15 and $30 \mathrm{~nm}[10,11]$. Unlike the apoptosome
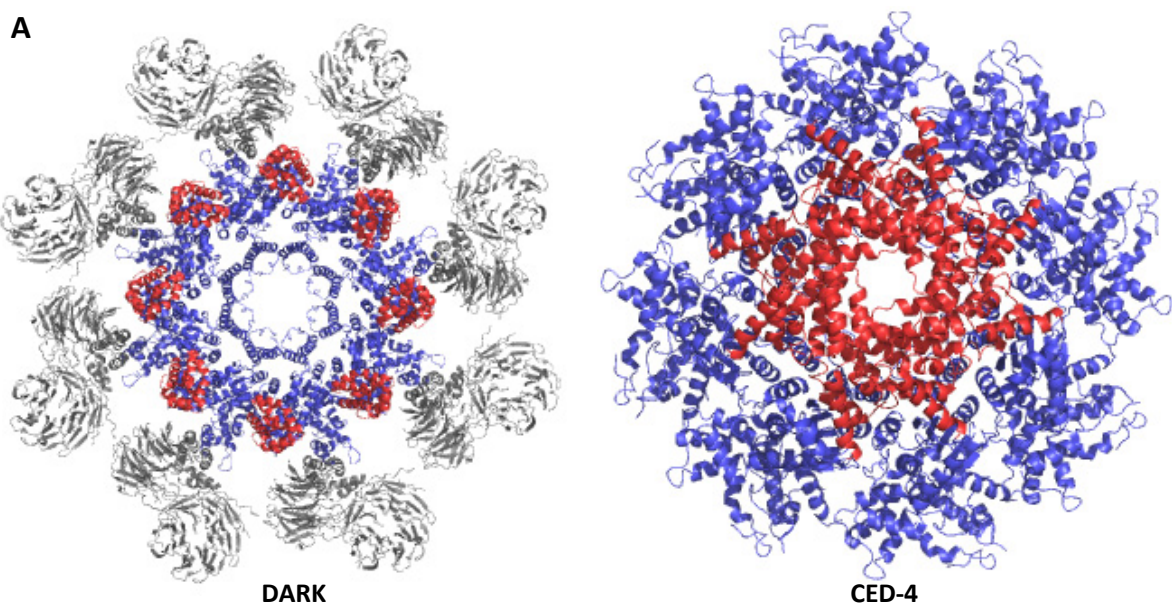

Wheel-like apoptosome structures

B
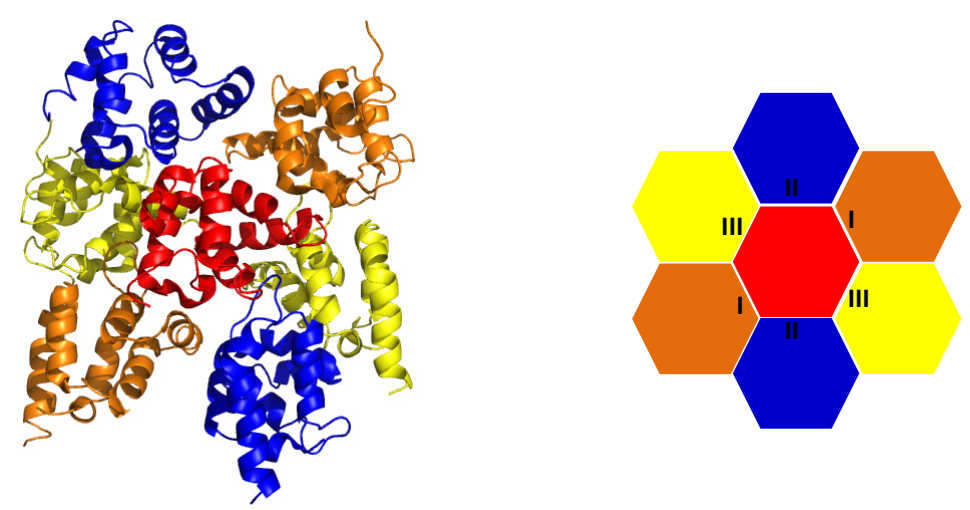

Classical death domain interactions as seen in the Myddosome

Figure 2: Structural and schematic representations of death domain superfamily complexes. (A) Orthologues of Apaf-1 from Drosophila melanogaster and Caenorhabditis elegans form wheel-like apoptosomes. Structure of a single-ring, DARK apoptosome showing the CARDs resting on top of an octomeric ring of NB-ARC domains, surrounded by WD40 domains (left panel; adapted from PDB 1VT4). Model of an octomeric CED4 apoptosome showing the CARDs forming a disc above the NB-ARC domains (generated using four dimeric molecules (PDB 3LQR) superimposed on the DARK apoptosome octomer (PDB 1VT4) (right panel). (B) The structural (left panel) and schematic (right panel) organisation of seven death domains taken from the MyD88 crystal structure (Myddosome). The central death domain (red) is surrounded by six others: those in orange bind through type I interactions, those in blue bind through type II interactions and those in yellow bind through type III interactions. The type of interface is labelled on the schematic representation. 
the stoichiometry of these "inflammasomes" is less well defined, consisting of either 5 to 7 (NLRP1) or, 11 or 12 (NLRC4/NAIP) protomers each. This variability may explain why these structures have yet to be successfully crystallised, and as yet their physiological relevance cannot be confirmed. However, recent molecular modelling studies have demonstrated that the CARD of ASC would be able to adopt a conformation compatible with such structural arrangements [12]. Other, noninflammasome forming NLR proteins, such as NLRC5, have been successfully modelled onto a heptameric apoptosome framework [13]. Whether the inflammasome forms such a complex within the cell, or indeed if it does how these then assemble to ultimately create a superstructure with a diameter more than 30-times that of an individual wheel remains to be determined.

In addition to the comparisons made to the apoptosome, macromolecular structures from the death domain (DD) superfamily, such as the PIDD (p53-induced death domain protein)-osome [14] and Myddosome [15], have helped drive mutagenic studies investigating the protein-protein interactions involved in inflammasome formation (Figure 2B). Although the components of the inflammasome do not contain death domains, they do possess Pyrin (PYD) and CARD motifs which are members of the DD superfamily. The PIDDosome and Myddosome structures have allowed three separate interaction interfaces (Types I, II and III) to be described. The interfaces function as key sites of contact in the protein-protein interactions required for assembly of the macromolecular complex. Collectively, these interfaces allow each death domain to interact with six others, mediating the formation of macromolecular structures (Figure 2B). The behaviour, role and importance of these interfaces is neatly reviewed in a recent paper by Kersse and Vandenabeele [16]. Consistent with a similar method of interaction disruption of the proposed interface residues in the PYD and CARD domains of the inflammasome has enabled the identification of key functional residues involved in complex formation. These include: a hydrophobic patch on the AIM2 PYD [17]; residues R10, D27, E41, K42, R55 and D59 of the caspase-1 CARD [18,19]; E14, R42, R75 and R80 in the Pyrin PYD; and multiple amino acids in both the PYD (E13, K21, R41, D48, D51) and CARD (R125, E130, D134, Y137, E144, R160, D191) of ASC [19,20]. Without question it has become apparent that the key inflammasome forming protein, ASC, uses more than one protein interface to engage more than one binding partner simultaneously during inflammasome formation [19-21]

\section{Molecular structures of inflamma- some components}

The molecular structure of an increasing number of the components of the inflammasome has been resolved. These include: the PYD of NLRP1 (PDB 1PN5), NLRP3 (PDB 3QF2), AIM2 (PDBs 407Q, 3VD8), ASC (PDBs 1UCP, 3J63) and Pyrin (PDB 2MPC) [17,20,22-26]; the CARD of NLRP1 (PDBs 4IFP, 3KAT) [27]; full-length ASC (PDB 2KN6) [28]; the auto-inhibited NLRC4 NBD (nucleotide-binding domain) and leucine rich repeats (LRR) (PDB 4KXF) [29]; the LRRs of NLRP1 (PDB 4IM6) [30]; and the HIN (hematopoietic interferon-inducible nuclear localization) domain of AIM2 in complex with dsDNA (PDBs 3RN2, 3RN5, 4JBM) [31,32]. Many structures of caspase-1 exist but they lack the CARD and so are not relevant to the mechanics of inflammasome assembly.

The isolated nature of the majority of these structures means that they can only provide a limited insight into the mechanisms of inflammasome assembly and the nature of the intra-molecular contacts involved. Although the PYD and CARD structures do provide insight into the residues likely to form binding interfaces which can then be investigated by mutagenesis studies. Until the inherent problem of protein aggregation can be reliably overcome this remains a significant hurdle in meeting the challenge of obtaining structural insight in to the protein-protein complexes formed in the inflammasome.

The structures of autoinhibited NLRC4 and AIM2 in complex with dsDNA have however been vital in helping our understanding of inflammasome regulation [29,32,32] (Figure 3). For example, NLRC4 is maintained in an inactive conformation through the formation of an ADP-dependent interaction between Helical Domain 2 and a single alpha helix in the NBD. Interactions between one surface of the NBD and the LRRs serve to lock the conformation, until it is presumably released following recognition of ligand by a NAIP protein and their subsequent interaction with NLRC4 [29] (Figure 3A). Meanwhile activation of AIM2 by dsDNA involves direct interaction between the ligand and the HIN domain of the receptor [31,32] (Figure 3B). Interestingly short DNA sequences are lined up along the binding surfaces to potentially mimic a longer sequence and recreate the presence of major and minor grooves. This is important for inhibition of AIM2 signalling in the mouse via the action of the protein p202. Although p202 competes with AIM2 to bind DNA, it arranges its HIN domains in a different orientation to AIM2 and could feasibly intercalate into the minor groove of AIM2-bound DNA to inhibit subsequent AIM2 oligomerisation and 


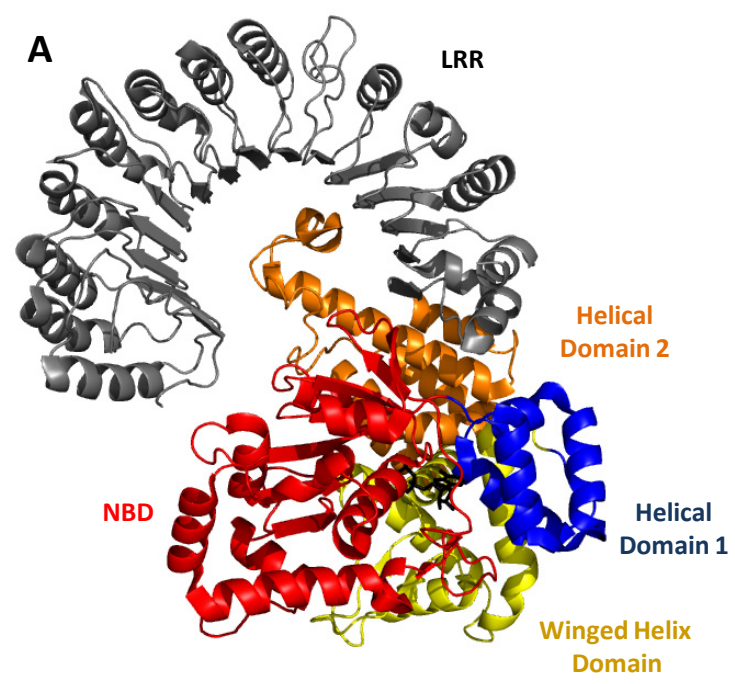

Autoinhibited NLRC4

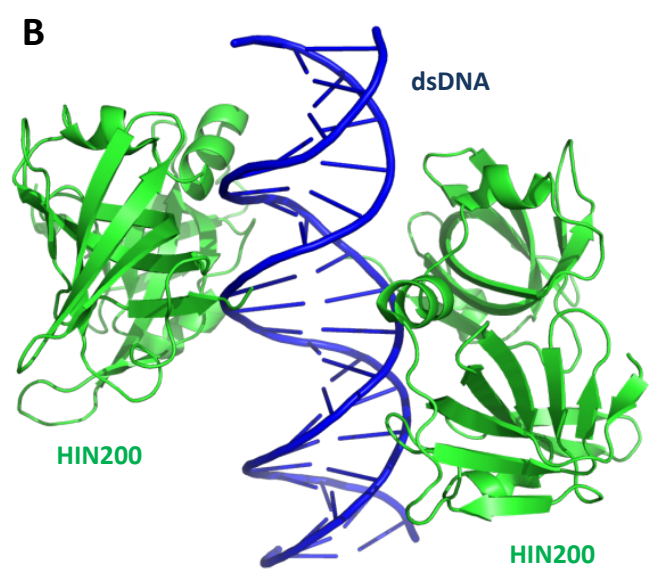

AIM2 HIN200 bound dsDNA

Figure 3: Inflammasome related structures. (A) The crystal structure of the NBD-LRR structure of NLRC4 (PDB 4KXF) in the autoinhibited conformation is shown, coloured by its subdomains: Red - NBD; Blue - Helical Domain 1; Yellow - Winged Helix Domain; Orange - Helical Domain 2; Grey - LRR; Black - ADP. (B) Crystal structure of the AIM2 HIN200 domains (green) bound to dsDNA (blue) (PDB 3RN2).

inflammasome formation [32]. Interestingly the structure of the NLRP1 LRRs [30] suggests that muramyl dipeptide, a potential NLRP1 ligand, is not capable of binding the LRRs. Whether this is indicative of an alternative binding site, or suggests that muramyl dipeptide is not in fact an activating ligand for NLRP1 remains to be determined.

\section{The rise of the filament}

An interesting twist on the classical view of inflammasome assembly occurs through the recent association of ASC filament formation and inflammasome activation. Two concurrent studies published earlier this year in Cell both reported that ASC could form filamentous structures $[25,33]$. Cai and co-workers demonstrated that the PYD of ASC has biochemical and genetic properties suggestive of prion-like behaviour and the formation of protein filaments (Figure 4A). Furthermore these filaments of ASC PYD were able to catalyse native ASC to form filamentous aggregates [33]. Meanwhile, using recombinant proteins $\mathrm{Lu}$ and colleagues demonstrated that both the AIM2 and NLRP3 PYD domains can act as nucleation points to initiate the formation of ASC-PYD filaments. The AIM2 PYD alone was sufficient to mediate this effect, however, NLRP3 mediated formation also required the NBD, suggesting that the process of NLRP3 NBD-mediated oligomerisaton plays an important role in the initiating steps that result in ASC-PYD filament formation, and that the precise mechanism of AIM2 and NLRP3 initiated inflammasome formation may differ [25].

Cryo-EM observations of the ASC-PYD filaments revealed a ring-like structure formed from three intertwined helical strands. The inner and outer diameters of this structure were about 2 and $9 \mathrm{~nm}$ respectively, much, much, smaller than the specks observed within cells, which appeared to be the result of filament aggregation. Formation of the filament could be disrupted by point mutations in residues predicted to mediate PYD-PYD interactions. In vitro reconstitution studies using fusion proteins of full-length ASC, the caspase-1 CARD, and the AIM2 PYD led to formation of star-like structures in which the caspase-1 CARD formed additional filaments extending outwards from a central focus of AIM2 and ASC (Figure 4B). EM of inflammasomes purified from THP1 cells, as well as immunogold EM of Cos-1 cells overexpressing ASC fused to eGFP resulted in the visualisation of large, relatively disordered filament containing structures [25].

Further support for the formation of aggregated fibres of ASC has been provided by back-to-back papers in Nature Immunology from the laboratories of Pelegrin and Latz [34,35]. Whilst characterising the inflammatory nature of inflammasome components released into the extracellular space both groups visualised the formation of ASC fibrils. Specks formed from ASC-mCerulean and released from cells were also able to seed the formation 
A

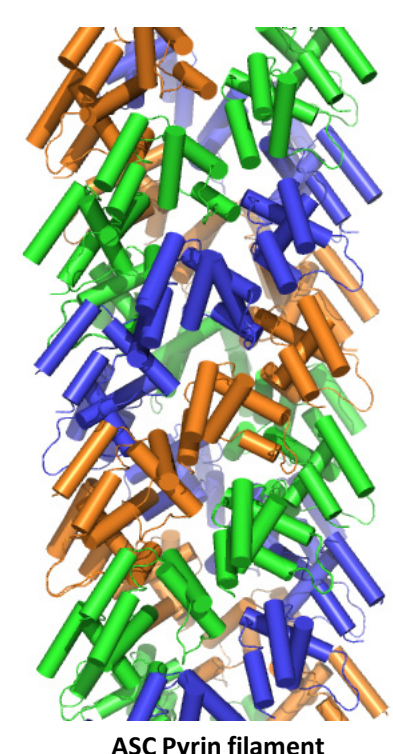

B

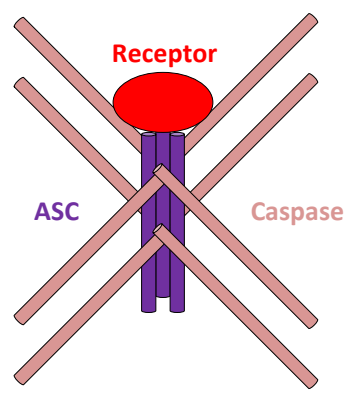

Filamentous structure

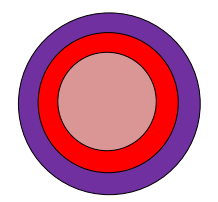

Endogenous ring structure
C

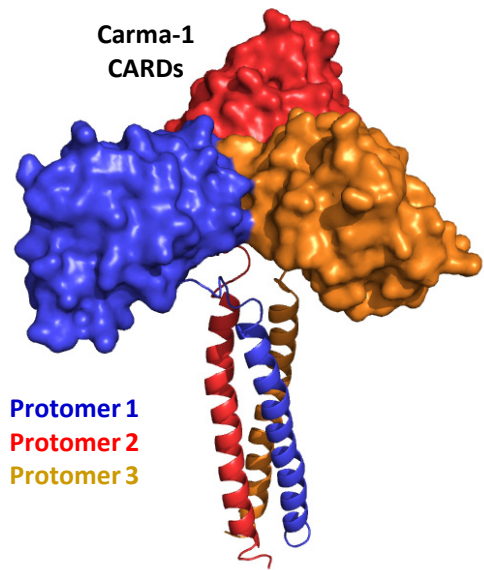

D

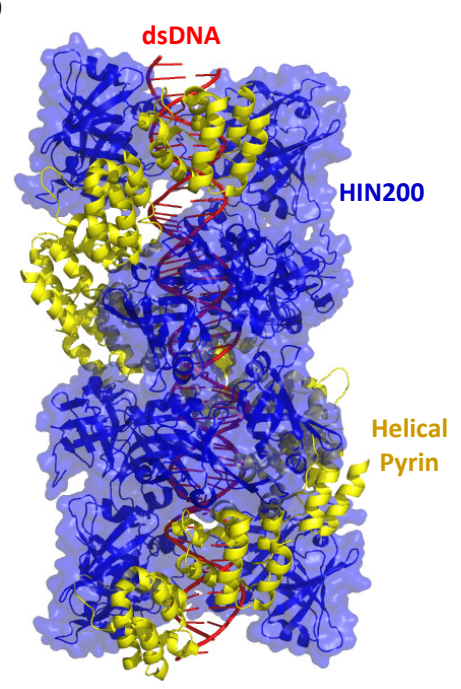

E

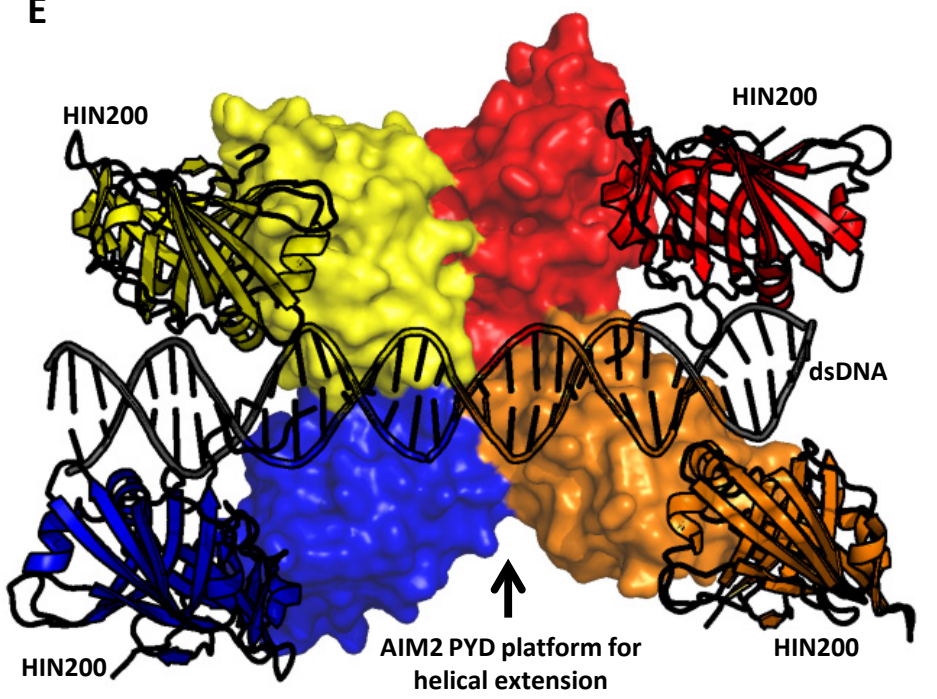

Figure 4: Helical filaments and the initiation of inflammasome formation. (A) Cylinder representation of the three membered helix formed by the ASC PYD (adapted from PDB 3J63). Each helical chain is coloured orange, green or blue. (B) Schematic organisation for an inflammasome formed from helical filaments (top) or as visualised endogenously (bottom). Inflammasome initiating receptors (red) act as a nucleation signal for speck formation. In overexpression systems this results in ASC filament formation (dark purple) and filamentous recruitment of caspases (light purple). With endogenous expression levels a concentric ring-like structure is formed. (C) Model for the formation of a CARMA1 CARD initiating helical structure mediated by the formation of a triple coiled-coil domain. Each CARMA1 protomer is coloured red, orange or blue. (D and E) Potential models for the formation of a helical PYD filaments by AIM2-like receptors. (D) HIN200 domains (blue) bind to DNA and allow the cooperative formation of a helical strand (yellow) around the DNA molecule (E) HIN200 domains from AIM2 or IFI16 (cartoon) bind to DNA and allow the cooperative formation of a platform for helical extension (surface).

of ASC-mCherry specks in recipient cells in a manner analogous to prions [35]. Interestingly these works were able to utilise EM to visualise ASC filaments in wild-type macrophages as well as in cells over-expressing ASC $[34,35]$. The observation that ASC can form filaments is however not new and overexpression of full-length ASC, and both the ASC CARD and PYD domains, have all been shown previously to form filaments in COS-7 cells [36]. In addition reconciliation of the formation of PYD-only filaments in the context of full-length ASC is needed, as is consolidation with the reported essential requirement for the ASC CARD in mediating speck formation [19]. 


\subsection{A wider perspective on filament formation}

The assembly of polymeric filaments which elute in the void volume during size exclusion purification is a common theme in recent studies of CARDs and PYDs. While structures based on the assembly of the Bcl-10 (B-cell lymphoma/leukemia 10) CARD [37], the MAVS (Mitochondrial antiviral-signaling protein) CARD [38] and the ASC PYD [25] are all remarkably different, they are each reported to be built up of interactions resembling the Type I, II and III interactions seen in DD complexes $[14,15]$. Spontaneous filament formation is limited for the individual domains but polymerisation can be induced by the introduction of known upstream binding partners.

This phenomenon has perhaps been best characterised in the case of the MAVS CARD, which can be induced to polymerise by the tandem CARDs (2CARD) from RIG-I (Retinoic acid inducible gene I). An initial crystallography study showed 2CARD forming a helical tetramer in the presence of ubiquitin and suggested that MAVS CARDs might polymerise by extending this helix [39]. The same group subsequently used a 2CARD-MAVS fusion construct to show the formation of a helical layer above 2CARD consisting of four MAVS CARDs [38]. In this case, the MAVS CARDs were mutated to allow their interaction with 2CARD but prevent filamentation. This study also produced an EM-derived structure of the MAVS filament which overlays well onto the RIG-I/MAVS crystal structure, suggesting that helix formation continues in the same manner as laid out by the initiator helix. The relationship between the RIG-I and MAVS CARDs provides the first structural evidence of an initiator helix forming and inducing filament formation along the same helical pattern. This concept gives rise to various lines of investigation regarding its relevance and application to other complexes based on death domain assemblies, as discussed below.

Polymerisation of the Bcl-10 CARD alone is limited but can be significantly enhanced by CARMA1 (CARDcontaining MAGUK protein 1), which residues at one end of nascent polymers [37]. Bcl-10 and CARMA1 interact via their CARDs but the monomeric CARMA1 CARD is in itself unable to induce Bcl-10 polymerisation. However, CARMA1 constructs including the coiled coil domains can form trimers or oligomers and these constructs are able to enhance Bcl-10 polymerisation [37]. With reference to the RIG-I/MAVS studies, a likely model is that CARMA1 oligomerisation through its coiled coil domains allows the stable assembly of an initiator helix between its CARDs, which is extended by Bcl-10 CARDs (Figure 4C).
Likewise, the ASC PYD alone shows limited polymerisation but this can be enhanced by using the AIM2 PYD or NLRP3 PYD-NBD as initiators ([25], see above). These models centre around the formation of a three-stranded helix built from ASC PYDs which interact in a similar way to classical death domain complexes. AIM2 and NLRP3 act as nucleation points for ASC elongation, likely by forming initiator helices from their own PYDs. The CARDs of ASC are found on the outside of the helix and interact with the CARD of caspase-1, appearing to initiate filaments of caspase-1 CARDs, extending away from the ASC helix. This results in the activation of caspase- 1 and the subsequent processing of IL-1 $\beta$ [25] (Figure 4B).

AIM2, like other AIM2-like receptors, is activated through the interaction of its HIN200 domain with dsDNA and a combination of dsDNA and AIM2 induced ASC polymerisation more efficiently than AIM2 alone [25]. The binding of the related AIM2-like receptor, gammainterferon-inducible protein 16 (IFI16), to dsDNA is a cooperative process where the DNA binding is dependent on the HIN200 domains while the cooperativity requires the PYD [40]. This has been explained by the formation of a signalling platform by the PYDs, which may resemble the ASC helix. The inner diameter of the ASC helix leaves open the possibilities that AIM2/IFI16 initiator helices may form in a bundle alongside the DNA molecule or around the DNA itself (Figure 4D \& E).

NLRP3 is autoinhibited by its LRR domain and removal of the LRR domain mimics receptor activation. As expected, $\triangle$ LRR NLRP3 was better at inducing ASC polymerisation than full-length NLRP3 [25]. EM studies of NLRP1 [10] and NLRC4/NAIP [11] show that these NLRs have the capacity to form circular wheel-like oligomers, similar to the apoptosome structures in which the central, nucleotidebinding domains mediate oligomerisation of the receptors while the CARDs or PYDs form a so far uncharacterised structure on top of this ring. As mentioned above the NLRP1 and NAIP/NLRC4 structures contain different numbers of protomers (5-7 and 11 or 12 respectively) [10,11]. Similarly, the human apoptosome is heptameric [41] while its homologues from C. elegans [42] and D. melanogaster [43] appear to be octomeric (Figure 2A \& B). In all of these cases, the instigation of signalling through the formation of an initiator helix at the centre of the ring structure offers a resolution that would allow variation in the oligomeric number of the receptor (Figure 5).

The new-found role of ASC in forming filaments may shed light on its role in NLRC4 signalling. Previously ASC was seen as a simple linker that connected the PYD from an NLRP or AIM2-like receptor to the CARD of caspase-1. Its role was therefore unclear in the case of NLRC4, which 

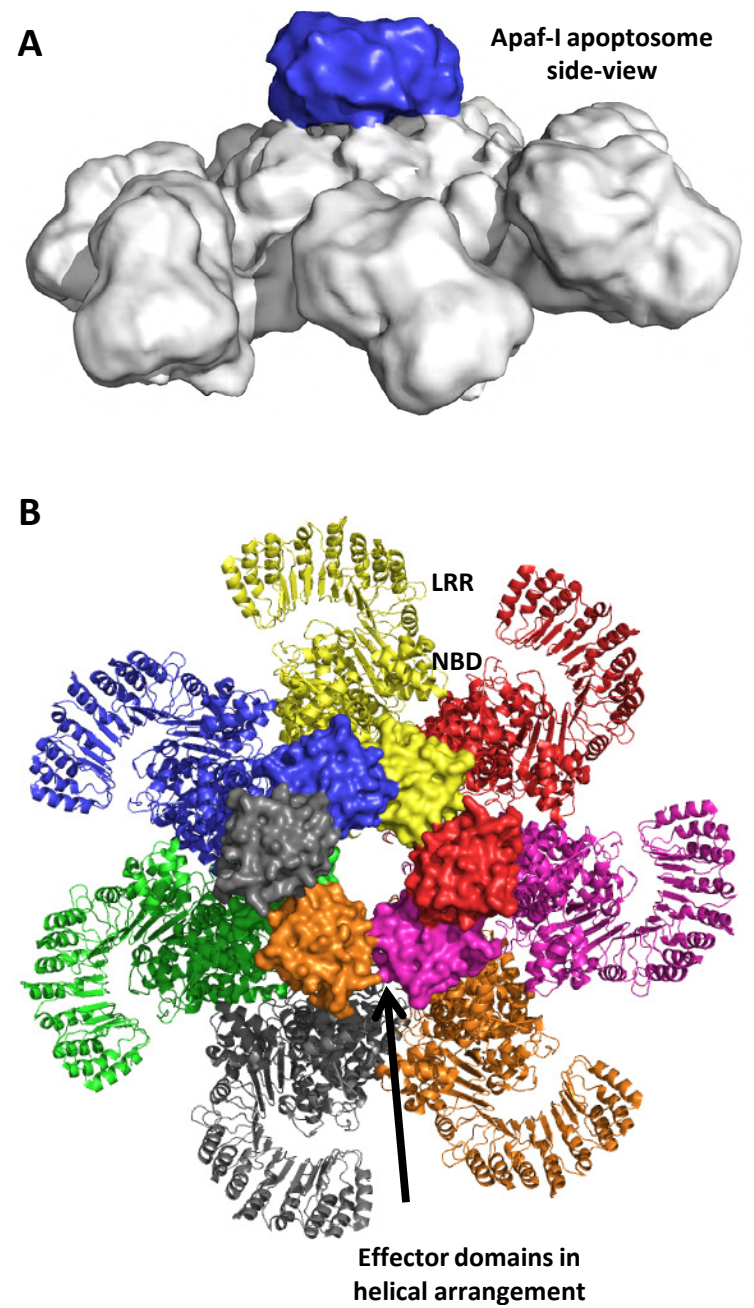

Figure 5: Mechanisms of potential initiator helix formation. (A) Side view of the electron density map of the Apaf-1 apoptosome suggests a slight helical twist, which could undergo propagation. (B) Theoretical model of the nucleating inflammasome structure demonstrating the feasibility of the effector domains (CARDs or PYDs) forming a helical complex after NBD mediated oligomerisation. In this heptameric conformation each protomer contributes an effector domain to a central initiator helix.

can already interact with caspase-1 through its CARD $[19,44]$ but requires ASC for high levels of IL-1 13 processing [45,46]. The induced polymerisation of ASC by NLRC4 is a potential mechanism for the increased caspase-1 activity.

In light of the seemingly common nature of filament formation it has recently been proposed that such filamentous structures represent a new paradigm in the formation of macromolecular signalling complexes [47]. However, regardless of how impressive these studies appear there remains the caveat that they are reliant on various combinations of protein overexpression, the use of recombinant fusion proteins, the presence of fluorescent tags, and protein purification or sample preparation approaches of varying severity. It remains unclear how exactly these may affect protein-protein interactions. Indeed proteins from the death domain superfamily are inherently prone to aggregation. The open-ended nature of the filaments described differs significantly from the helical structures of the PIDDosome and MyDDosome, in which the stoichiometry is well defined and the structural assembly finite $[14,15]$. Further investigation is needed to confirm whether these filaments are physiologically relevant or an artefact of the high levels of protein achieved in overexpression and recombinant systems.

\subsection{The ultrastructure of the inflamamsome}

The use of fluorescent ASC-fusion proteins or the incorporation of fluorescent caspase inhibitors and substrates has enabled visualisation of inflammasome speck formation to become a routine read out in cellbased studies [48]. As well as providing a ready marker for 
inflammasome activation these techniques highlight the large size of the speck, with over-expressed fluorescent ASC-fusions routinely producing specks of up to two microns in diameter. The overall dimensions of a speck are not fixed, they presumably relate to, and are regulated by, the availability of ASC, and other components, within the cell. Consequently the higher the level of ASC, the bigger the speck can become. Specks originating from overexpression systems usually appear solid and will often be seen to have 'hair-like' protrusions which have been proposed to represent sites of active ASC recruitment [35]. This would correlate strongly with the EM-observed string-like blobs upon ASC overexpression and the proposed process of filament formation [25].

Although ASC fibrils have been reported in wildtype cells [34,35] the visualisation of endogenous inflammasomes has on other occasions painted a slightly different picture. The first observations of ASC complexes by pre-embedding EM occurred prior to the discovery of the inflammasome [49]. HL-60 cells were cultured with retinoic acid to induce apoptosis and ASC clustering was observed at the periphery of the cells. These clusters had hollow centres. Early images of endogenous levels of ASC [50] also presented with hollow centres in contrast to the solid speckles and filament-like aggregates observed with overexpressed ASC [36]. More recently Man and colleagues have utilised Bayesian localisation super-resolution microscopy to visualise the endogenous inflammasomes formed in response to Salmonella infection [51]. Using both immunolabelling and fluorescent-labelled inhibitor of caspases staining they observed hollow ring-like structures, with maximum diameters of approximately 0.6 to 0.7 microns. These inflammasome rings were organised in a concentric manner consisting of an outer ASC ring, a central NLR-containing ring consisting of both NLRC4 and NLRP3, and an internal caspase containing ring. An organisation markedly different to the star-like filament conformation reported by EM [25] (Figure 4B) and one that raises questions regarding the potential differences in macromolecular structure formed under endogenous conditions, compared to those requiring enhanced levels of protein expression.

One must also consider the possibility that the inflammasome is capable of forming multiple functional structures within the cell. For example, both the wheellike apoptosome-like structures and the specks may exist as discrete entities within the cell and fulfil specific functional roles. To our knowledge the apoptosome has never been visualised in the cell in a manner analogous to the inflammasome, but the functional relevance of the structures observed has not been questioned. One possibility is that a helical wheel structure could act to nucleate speck formation. Alternatively, they may act as speck-independent platforms for caspase-1 activation and pro-IL-1 $\beta$ processing. Support for this latter theory is provided by the heightened production of IL-1 $\beta$ by macrophages of patients with cryopyrin-associated periodic syndromes due to activating mutations in NLRP3 [50,52]. This increase occurs in the absence of both inflammasome stimulation and speck formation.

\subsection{The inflammasome in the future}

The speck has been described as being formed of numerous interwoven filaments forming a larger structure, analogous to a bird's nest [53]. Following nucleation, the whole cellular complement of ASC appears to be drawn to the speck. As such, the ratio of nucleating centres to ASC molecules will determine the length of resulting filaments. Experiments in which the levels of ASC and receptor are varied would help provide confirmation of this theory as overexpression of ASC should yield longer filaments and overexpression of a receptor should result in shorter filaments. Of course other factors such as Pyrin-Only-Proteins may also affect the length of PYDdriven filaments, and it will be interesting to see their influence evaluated through the development and use of Pyrin-Only-Protein knock out cells. The possibility that filaments of different length will associate to form distinct speck structures makes studies observing endogenous levels of protein especially valuable.

Assuming that the role of receptors is to initiate ASC filamentation for speck assembly, results in a number of interesting questions. For example, how much order or disorder is there in the formation of specks from filaments? What roles do the ASC PYD and CARD play in filamentation and inter-filament binding? Do receptors remain attached to extended filaments indefinitely or can they dissociate and initiate new filaments?

Models of the speck will have to take into account the distinct spatial arrangement of different receptor and caspase proteins around the ASC ring. For example, Man et al. show that the NLRC4 and NLRP3 receptors in an endogenous speck form distinct regions [51]. Is this due to a difference in the way the receptors bind to ASC or is it due to sequential recruitment to the growing speck? Analysis of speck formation in real-time could revolutionise our understanding of the dynamics of speck formation.

Notwithstanding the recent advances in our understanding of the organisation, the composition, and the possible assembly mechanisms of the inflammasome there is much still to beaddressed. For example our knowledge of both 
the spatial and temporal pathways involved in inflammasome formation remains limited. Careful use of knock out cells and visualisation of the endogenous inflammasome could help address these. Meanwhile, further information about the composition and potential stoichiometric requirements of the inflammasome is needed. This could be addressed using mass spectroscopy as has recently been achieved in stoichiometric studies of the death-inducing signalling complex [54]. Whilst, we know that speck formation can be extremely rapid questions remain unanswered about the dynamics of protein recruitment and would benefit from the use of carefully kinetic experiments. Finally, we still need to resolve the reasons underpinning the different appearances of the inflammasome specks visualised to date. This may well require application of various technologies to multiple systems to look for consistency. Certainly applying the highresolution techniques used to visualise the endogenous inflammasome to overexpression systems could rapidly clarify whether or not the global architecture of endogenous and exogenous inflammasomes differs. This is an exciting time to be working in this research field and without question the future holds not only the answers to the current areas of uncertainty, but no doubt some more surprises too.

Acknowledgements: The authors have no conflicts of interest to declare. We would like to thank Professor Clare Bryant and members of her lab for helpful discussion. This work was funded by a Wellcome Trust Career Development Fellowship to TPM (WT085090MA ).

\section{Abbreviations:}

AIM2, Absent in Melanoma 2; Apaf-1, Apoptotic proteaseactivating factor 1; ASC, apoptosis-associated speck-like protein containing a CARD; Bcl-10, B-cell lymphoma/ leukemia 10; CARD, caspase activation and recruitment domain; CARMA1, CARD-containing MAGUK protein 1; DD, Death Domain; EM, electron microscopy; HIN, hemopoietic expression, interferon-inducibility, nuclear localization; IFI16, gamma-interferon-inducible protein 16; LRR, leucine-rich repeat; MAVS, Mitochondrial antiviral-signaling protein; NAIP, neuronal apoptosis inhibitory protein; NBD, NBD, nucleotide-binding domain; NLR, nucleotide-binding domain, and leucine-rich repeat containing receptor; NLRC, NLR family, CARD domain containing; NLRP, NLR family, pyrin domain containing; PIDD, p53-induced death domain protein; PYD, Pyrin Domain; RIG-I, Retinoic acid-inducible gene I.

\section{References}

[1] Salvesen GS., Dixit VM., Caspase activation: the inducedproximity model., Proc. Natl. Acad. Sci. U. S. A., 1999, 96, 10964-7

[2] Lamkanfi M., Dixit VM., Mechanisms and functions of inflammasomes., Cell., Elsevier Inc.; 2014, 157, 1013-22

[3] Wen H., Miao EA., Ting JP-Y., Mechanisms of NOD-like receptorassociated inflammasome activation., Immunity., Elsevier Inc.;, 2013, 39, 432-41

[4] Latz E., Xiao TS., Stutz A., Activation and regulation of the inflammasomes, Nat. Rev. Immunol., 2013, 13, 397-411

[5] Cheng J., Waite AL., Tkaczyk ER., Ke K., Richards N., Hunt AJ., et al., Kinetic properties of ASC protein aggregation in epithelial cells., J. Cell. Physiol., 2010, 222, 738-47

[6] Case CL., Roy CR., Asc modulates the function of NLRC4 in response to infection of macrophages by Legionella pneumophila., MBio., 2011, 2, e00117-11

[7] Zhong Y., Kinio A., Saleh M., Functions of NOD-Like Receptors in Human Diseases., Front. Immunol., 2013, 4, 333

[8] Dinarello CA., van der Meer JWM., Treating inflammation by blocking interleukin-1 in humans., Semin. Immunol., Elsevier Ltd;, 2013, 25, 469-84

[9] Acehan D., Jiang X., Morgan DG., Heuser JE., Wang X., Akey CW., Three-dimensional structure of the apoptosome: implications for assembly, procaspase- 9 binding, and activation., Mol. Cell., 2002, 9, 423-32

[10] Faustin B., Lartigue L., Bruey J., Luciano F., Sergienko E., BaillyMaitre B., et al., Reconstituted NALP1 inflammasome reveals two-step mechanism of caspase-1 activation., Mol. Cell., 2007, $25,713-24$

[11] Halff EF., Diebolder CA., Versteeg M., Schouten A., Brondijk THC., Huizinga EG., Formation and structure of a NAIP5-NLRC4 inflammasome induced by direct interactions with conserved $\mathrm{N}$ - and C-terminal regions of flagellin., J. Biol. Chem., 2012, 287, 38460-72

[12] Alba E de., Santiveri CM., Oroz J., A ring-like model for ASC self-association via the CARD domain, Inflammasome., 2014, 1, 1639-41

[13] Mótyán JA., Bagossi P., Benkő S., Tózsér J., A molecular model of the full-length human NOD-like receptor family CARD domain containing 5 (NLRC5) protein., BMC Bioinformatics., 2013, 14, 275

[14] Park HH., Logette E., Raunser S., Cuenin S., Walz T., Tschopp J., et al., Death domain assembly mechanism revealed by crystal structure of the oligomeric PIDDosome core complex., Cell., 2007, 128, 533-46

[15] Lin S-C., Lo Y-C., Wu H., Helical assembly in the MyD88-IRAK4-IRAK2 complex in TLR/IL-1R signalling., Nature., 2010, 465, 885-90

[16] Kersse K., Verspurten J., Vanden Berghe T., Vandenabeele P., The death-fold superfamily of homotypic interaction motifs., Trends Biochem. Sci., Elsevier Ltd;, 2011, 36, 541-52

[17] Jin T., Perry A., Smith P., Jiang J., Xiao TS., Structure of the absent in melanoma 2 (AIM2) pyrin domain provides insights into the mechanisms of AIM2 autoinhibition and inflammasome assembly., J. Biol. Chem., 2013, 288, 13225-35

[18] Kersse K., Lamkanfi M., Bertrand MJM., Vanden Berghe T., Vandenabeele $P$., Interaction patches of procaspase-1 caspase 
recruitment domains (CARDs) are differently involved in procaspase- 1 activation and receptor-interacting protein 2 (RIP2)-dependent nuclear factor KB signaling., J. Biol. Chem., 2011, 286, 35874-82

[19] Proell M., Gerlic M., Mace PD., Reed JC., Riedl SJ., The CARD plays a critical role in ASC foci formation and inflammasome signalling., Biochem. J., 2013, 449, 613-21

[20] Vajjhala PR., Mirams RE., Hill JM., Multiple binding sites on the pyrin domain of ASC protein allow self-association and interaction with NLRP3 protein., J. Biol. Chem., 2012, 287, 41732-43

[21] Vajjhala PR., Kaiser S., Smith SJ., Ong Q-R., Soh SL., Stacey KJ., et al., Identification of multifaceted binding modes for pyrin and ASC pyrin domains gives insights into pyrin inflammasome assembly., J. Biol. Chem., 2014, 289, 23504-19

[22] Hiller S., Kohl A., Fiorito F., Herrmann T., Wider G., Tschopp J., et al., NMR Structure of the Apoptosis- and Inflammation-Related NALP1 Pyrin Domain, Structure., 2003, 11, 1199-205

[23] Bae JY., Park HH., Crystal structure of NALP3 protein pyrin domain (PYD) and its implications in inflammasome assembly., J. Biol. Chem., 2011, 286, 39528-36

[24] Lu A., Kabaleeswaran V., Fu T., Magupalli VG., Wu H., Crystal structure of the F27G AIM2 PYD mutant and similarities of its self-association to DED/DED interactions., J. Mol. Biol., Elsevier Ltd;, 2014, 426, 1420-7

[25] Lu A., Magupalli VG., Ruan J., Yin Q., Atianand MK., Vos MR., et al., Unified polymerization mechanism for the assembly of ASC-dependent inflammasomes., Cell., 2014, 156, 1193-206

[26] Liepinsh E., Barbals R., Dahl E., Sharipo A., Staub E., Otting G., The Death-domain Fold of the ASC PYRIN Domain, Presenting a Basis for PYRIN/PYRIN Recognition, J. Mol. Biol., 2003, 332, 1155-63

[27] Jin T., Curry J., Smith P., Jiang J., Xiao TS., Structure of the NLRP1 caspase recruitment domain suggests potential mechanisms for its association with procaspase-1., Proteins., 2013, 81, 1266-70

[28] De Alba E., Structure and interdomain dynamics of apoptosisassociated speck-like protein containing a CARD (ASC)., J. Biol. Chem., 2009, 284, 32932-41

[29] Hu Z., Yan C., Liu P., Huang Z., Ma R., Zhang C., et al., Crystal structure of NLRC4 reveals its autoinhibition mechanism., Science., 2013, 341, 172-5

[30] Reubold TF., Hahne G., Wohlgemuth S., Eschenburg S., Crystal structure of the leucine-rich repeat domain of the NOD-like receptor NLRP1: Implications for binding of muramyl dipeptide., FEBS Lett., Federation of European Biochemical Societies;, 2014, 588, 3327-32

[31] Jin T., Perry A., Jiang J., Smith P., Curry JA., Unterholzner L., et al., Structures of the HIN domain:DNA complexes reveal ligand binding and activation mechanisms of the AIM2 inflammasome and IFI16 receptor., Immunity., Elsevier Inc.;, 2012, 36, 561-71

[32] Ru H., Ni X., Zhao L., Crowley C., Ding W., Hung L-W., et al., Structural basis for termination of AIM2-mediated signaling by p202., Cell Res., 2013, 23, 855-8

[33] Cai X., Chen J., Xu H., Liu S., Jiang Q-X., Halfmann R., et al., Prion-like Polymerization Underlies Signal Transduction in Antiviral Immune Defense and Inflammasome Activation., Cell., Elsevier Inc.; 2014, 156, 1207-22

[34] Baroja-Mazo A., Martín-Sánchez F., Gomez Al., Martínez CM., Amores-Iniesta J., Compan V., et al., The NLRP3 inflammasome is released as a particulate danger signal that amplifies the inflammatory response., Nat. Immunol., 2014, 15, 738-48

[35] Franklin BS., Bossaller L., De Nardo D., Ratter JM., Stutz A., Engels G., et al., The adaptor ASC has extracellular and "prionoid" activities that propagate inflammation., Nat. Immunol., 2014, 15, 727-37

[36] Masumoto J., Taniguchi S., Sagara J., Pyrin N-terminal homology domain- and caspase recruitment domaindependent oligomerization of ASC., Biochem. Biophys. Res. Commun., 2001, 280, 652-5

[37] Qiao Q., Yang C., Zheng C., Fontán L., David L., Yu X., et al., Structural Architecture of the CARMA1/Bcl10/MALT1 Signalosome: Nucleation-Induced Filamentous Assembly, Mol. Cell., 2013, 51, 766-79

[38] Wu B., Peisley A., Tetrault D., Li Z., Egelman EH., Magor KE., et al., Molecular Imprinting as a Signal-Activation Mechanism of the Viral RNA Sensor RIG-I, Mol. Cell., Elsevier Inc.;, 2014, 55, 511-23

[39] Peisley A., Wu B., Xu H., Chen ZJ., Hur S., Structural basis for ubiquitin-mediated antiviral signal activation by RIG-I., Nature., Nature Publishing Group;, 2014, 509, 110-4

[40] Morrone SR., Wang T., Constantoulakis LM., Hooy RM., Delannoy MJ., Sohn J., Cooperative assembly of IFI16 filaments on dsDNA provides insights into host defense strategy., Proc. Natl. Acad. Sci. U. S. A., 2014, 111, E62-71

[41] Yuan S., Yu X., Asara JM., Heuser JE., Ludtke SJ., Akey CW., The holo-apoptosome: activation of procaspase- 9 and interactions with caspase-3., Structure., Elsevier Ltd;, 2011, 19, 1084-96

[42] Qi S., Pang Y., Hu Q., Liu Q., Li H., Zhou Y., et al., Crystal structure of the Caenorhabditis elegans apoptosome reveals an octameric assembly of CED-4., Cell., Elsevier Ltd;, 2010, 141, 446-57

[43] Yuan S., Yu X., Topf M., Dorstyn L., Kumar S., Ludtke SJ., et al., Structure of the Drosophila apoptosome at 6.9 A resolution., Structure., 2011, 19, 128-40

[44] Poyet JL., Srinivasula SM., Tnani M., Razmara M., FernandesAlnemri T., Alnemri ES., Identification of Ipaf, a human caspase-1-activating protein related to Apaf-1., J. Biol. Chem., 2001, 276, 28309-13

[45] Broz P., Newton K., Lamkanfi M., Mariathasan S., Dixit VM., Monack DM., Redundant roles for inflammasome receptors NLRP3 and NLRC4 in host defense against Salmonella., J. Exp. Med., 2010, 207, 1745-55

[46] Miao EA., Leaf IA., Treuting PM., Mao DP., Dors M., Sarkar A., et al., Caspase-1-induced pyroptosis is an innate immune effector mechanism against intracellular bacteria., Nat. Immunol., Nature Publishing Group;, 2010, 11, 1136-42

[47] Wu H., Higher-order assemblies in a new paradigm of signal transduction., Cell., Elsevier Inc.;, 2013, 153, 287-92

[48] Stutz A., Horvath GL., Monks BG., Latz E., ASC speck formation as a readout for inflammasome activation., Methods Mol. Biol., 2013, 1040, 91-101

[49] Masumoto J., Taniguchi S., Ayukawa K., Sarvotham H., Kishino T., Niikawa N., et al., ASC, a Novel 22-kDa Protein, Aggregates during Apoptosis of Human Promyelocytic Leukemia HL-60 Cells, J. Biol. Chem., 1999, 274, 33835-8

[50] Agostini L., Martinon F., Burns K., McDermott MF., Hawkins PN., Tschopp J., NALP3 forms an IL-1beta-processing inflammasome with increased activity in Muckle-Wells autoinflammatory disorder., Immunity., 2004, 20, 319-25 
[51] Man SM., Hopkins LJ., Nugent E., Cox S., Glück IM., Tourlomousis P., et al., Inflammasome activation causes dual recruitment of NLRC4 and NLRP3 to the same macromolecular complex., Proc. Natl. Acad. Sci. U. S. A., 2014, 111, 7403-8

[52] Aksentijevich I., Nowak M., Mallah M., Chae JJ., Watford WT., Hofmann SR., et al., De novo CIAS1 mutations, cytokine activation, and evidence for genetic heterogeneity in patients with neonatal-onset multisystem inflammatory disease (NOMID): a new member of the expanding family of pyrinassociated autoinflammatory diseases., Arthritis Rheum., 2002, 46, 3340-8
[53] Richards N., Schaner P., Diaz A., Stuckey J., Shelden E., Wadhwa A., et al., Interaction between pyrin and the apoptotic speck protein (ASC) modulates ASC-induced apoptosis., J. Biol. Chem., 2001, 276, 39320-9

[54] Dickens LS., Boyd RS., Jukes-Jones R., Hughes MA., Robinson GL., Fairall L., et al., A death effector domain chain DISC model reveals a crucial role for caspase- 8 chain assembly in mediating apoptotic cell death., Mol. Cell., Elsevier Inc.;, 2012, 47, 291-305 